\title{
Will There Be Energy Frontier Colliders After LHC?
}

\author{
Vladimir Shiltsev ${ }^{1}$ \\ Fermi National Accelerator Laboratory \\ PO Box 500, Batavia IL 60510, USA \\ E-mail: shiltsev@fnal.gov
}

High energy particle colliders have been in the forefront of particle physics for more than three decades. At present the near term US, European and international strategies of the particle physics community are centered on full exploitation of the physics potential of the Large Hadron Collider (LHC) through its high-luminosity upgrade (HL-LHC). The future of the world-wide HEP community critically depends on the feasibility of possible post-LHC colliders. The concept of the feasibility is complex and includes at least three factors: feasibility of energy, feasibility of luminosity and feasibility of cost. Here we overview all current options for post-LHC colliders from such perspective (ILC, CLIC, Muon Collider, plasma colliders, CEPC, FCC, HE-LHC) and discuss major challenges and accelerator $\mathrm{R} \& \mathrm{D}$ required to demonstrate feasibility of an energy frontier accelerator facility following the LHC. We conclude by taking a look into ultimate energy reach accelerators based on plasmas and crystals, and discussion on the perspectives for the far future of the accelerator-based particle physics.

38th International Conference on High Energy Physics

3-10 August 2016

Chicago, USA

\footnotetext{
${ }^{1}$ Fermi Research Alliance, LLC operates Fermilab under contract no. DE-AC0207CH11359 with the U.S. Department of Energy
} 


\section{Introduction}

Colliding beam facilities which produce high-energy collisions (interactions) between particles of approximately oppositely directed beams have been on the forefront of particle physics for more than half a century and twenty nine reached operational stage [1]. Their energy has been on average increasing by a factor of 10 every decade until about the mid-1990's. Notably, the hadron colliders were 10-20 times more powerful. Since then, following the demands of high energy physics (HEP), the paths of the colliders diverged: to reach record high energies in the particle reaction the Large Hadron Collider was built at CERN, while new $e+e$ - colliders called "particle factories" were focused on detailed exploration of phenomena at much lower energies. The Tevatron, LEP and HERA established the Standard Model of particle physics. The current landscape of the high energy physics is dominated by the LHC. The next generation of colliders is expected to lead the exploration of the smallest dimensions beyond the current Standard Model.

While the development of energy frontier colliders over the past five decades initiated a wide range of innovation in accelerator physics and technology which resulted in 100-fold increase in energy (for both hadron and lepton colliding facilities) and $10^{4}-10^{6}$ fold increase of the luminosity, the progress in the maximum c.o.m. energy has drastically slowed down since the early 1990's and the lepton colliders even went backwards in energy to study rare processes - see, e.g., Fig.1 in [2]. Moreover, the number of the colliding beam facilities in operation has dropped from 9 two decades ago to 7 now (2016). The future of accelerator-based HEP beyond LHC has been recently debated by several authors in $[2,3,4,5]$ and many technical details discussed in the collective book "Challenges and Goals for Accelerators in the XXI Century" [6]. Here we bring an economical (financial) perspective to the discussion on feasible colliders beyond the LHC and show that options based on traditional acceleration technologies are very much limited. Only “... technological quantum leaps...will drive the long-term progress of the field. We can expect that these ambitions and far sighted R7D programmes in accelerator technology will redefine the field of high-energy physics in the XXI century...” (M.Mangano, [6], p.21). In general, the discussion on the "beyond the LHC" energy frontier accelerators comes to the question of the right balance between the physics reach of the future facilities and their feasibility which usually assumes the feasibility of their energy reach (whether it is possible to reach the design c.o.m. energy), feasibility of the performance (how challenging is the declared design luminosity) and cost feasibility (is it affordable to build and operate?). While the first two criteria (energy and performance reach) are relatively easy to address on the base of the current state-of-the-art accelerator technology (of, e.g., normal- and superconducting magnets, RF, etc) and beam physics, the feasibility of the cost requires analysis of both the perspective available resources and the facility cost range.

In the analysis below we will use the cost of LHC - about 10B \$ at today's prices - as a reference for a globally affordable future facility and compare it with the resources required to build "beyond the LHC" colliders, including "near future" facilities with possible construction start within a decade - such as the international $e+e$ - linear collider in Japan (ILC) [7] and circular $e+e$ - colliders in China (CерC) [8] and Europe (FCC-ee) [9]; "future" colliders with construction start envisioned 10-20 years from now - such as linear $e+e$ - collider at CERN (CLIC) [10], muon collider [11], and circular hadron colliders in China (SppC) [8], Europe (HE-LHC [12] and FCCpp [9]) and USA (VLHC [13]); and an ultimate "far future" colliders with time horizon beyond the next two decades based on beam-plasma [14], laser-plasma [15] and crystal-plasma [16, 2] acceleration technologies. 


\section{Cost of large post-LHC accelerators}

All large accelerators built so far are based on four major acceleration technologies which employ either normal-conducting RF, or super-conducting RF, or magnets, again normal- or super-conducting. Some used more than one technology at once. All these technologies are well understood and their costs and potentials can be extrapolated from the past experience. In addition, construction of accelerators usually involves civil construction, often - tunneling, and creating of infrastructure, including high-power electric and cryogenic ones. All these technologies are commercial, in the sense that we just buy corresponding services from industries and, again, their costs are known pretty well. Therefore, one can expect that at least for future accelerators based on these "traditional" accelerator and infrustructure technologies, an extrapolation can be done and a rough cost estimate (better say - cost rage) can be obtained. Indeed, an analysis of the known costs of large accelerator facilities has been undertaken in [17]. Based on publicly available costs for 17 large accelerators of the past, present and those currently in the planning stage it was shown that the "total project cost (TPC)" (sometimes cited as "the US accounting") of a collider can be broken up into three major parts corresponding to "civil construction", "accelerator components", "site power infrastructure". The three respective cost components can be parameterized by just three parameters - the total length of the facility tunnels $L_{f}$, the center-ofmass or beam energy $E$, and the total required site power $P$ - and over almost 3 orders of magnitude of $L_{f}, 4.5$ orders of magnitude of $E$ and more than 2 orders of magnitude of $P$ the so-called " $\alpha \beta \gamma$ cost model" works with $\sim 30 \%$ accuracy [17]:

$$
\text { Total Project Cost } \approx \alpha \times(\text { Length } / 10 \mathrm{~km})^{1 / 2}+\beta \times(\text { Energy } / \text { TeV })^{1 / 2}+\gamma \times(\text { Power } / 100 M W)^{1 / 2} \text {, }
$$

where coefficients $\alpha=2 B \$ /(10 \mathrm{~km})^{1 / 2}, \gamma=2 B \$ /(100 \mathrm{MW})^{1 / 2}$, and accelerator technology dependent coefficient $\beta$ is equal to $10 \mathrm{~B} \$ / \mathrm{TeV}^{1 / 2}$ for superconducting RF accelerators, $8 \mathrm{~B} \$ / \mathrm{TeV}^{1 / 2}$ for normalconducting ("warm") RF, $1 B \$ / \mathrm{TeV}^{1 / 2}$ for normal-conducting magnets and $2 B \$ / T e V^{1 / 2}$ for SC magnets (all numbers in 2014 US dollars).

Let's take the LHC as an example. The first component of the " $\alpha \beta \gamma$-model” is the cost of some $40 \mathrm{~km}$ of LHC tunnels (including $27 \mathrm{~km}$ of the LEP tunnel, $7 \mathrm{~km}$ of SPS, injectors and beamlines) which can be estimated as $2 B \$ \times(40 / 10 \mathrm{~km})^{1 / 2}=4 B \$$. The estimate of the second component is dominated by the cost of SC magnets for $14 \mathrm{TeV}$ com collider, i.e., $2 B \$ \times(14)^{1 / 2}=7.5 B \$$. Finally, the estimate of the $150 \mathrm{MW}$ power infrustructure piece is $2 B \$ \times(150 \mathrm{MW} / 100 \mathrm{MW})^{1 / 2}=2.5 B \$$, that makes the TPC range of the LHC - if built from scratch - equal to $4 B \$+7.5 B \$+2.5 B \$=14 B \$$ $\pm 4.5 B \$$. The CERN LHC Factbook [18] indicates the cost of LHC project of 6.5BCHF, including 5BCHF for accelerator facility. These numbers are in so-called "European accounting"- the different methodology of the cost estimates widely used in Europe - that includes only the industrial contracts for major items like civil engineering, the accelerator elements and corresponding labor requirements (such approach is often referred). Usually, the "European accounting" is factor of 2.0-2.5 lower than the US DOE Office of Science's “the total project cost” (TPC) accounting [17] which additionally includes the costs of the required R\&D, development of the engineering design, project management, escalation, contingency, overhead funds, project-specific facility site development, sometimes - detectors, etc. Therefore, the TPC of the LHC accelerator project is some $5 B C H F \times(2-2.5)=10-12.5 B C H F=10-12.5 B \$$. Add an estimated 3-4B $\$$ for the LHC injector complex needed if the LHC would be built as a "green field" and one gets the LHC TPC of about $13-16.5 B \$$ - very much in line with the " $\alpha \beta \gamma$-estimate" we obtained above. 
Similar kind of estimates have been done many facilities currently considered for postLHC HEP accelerators - see, e.g, [19] - and summarized in Fig.1 below.

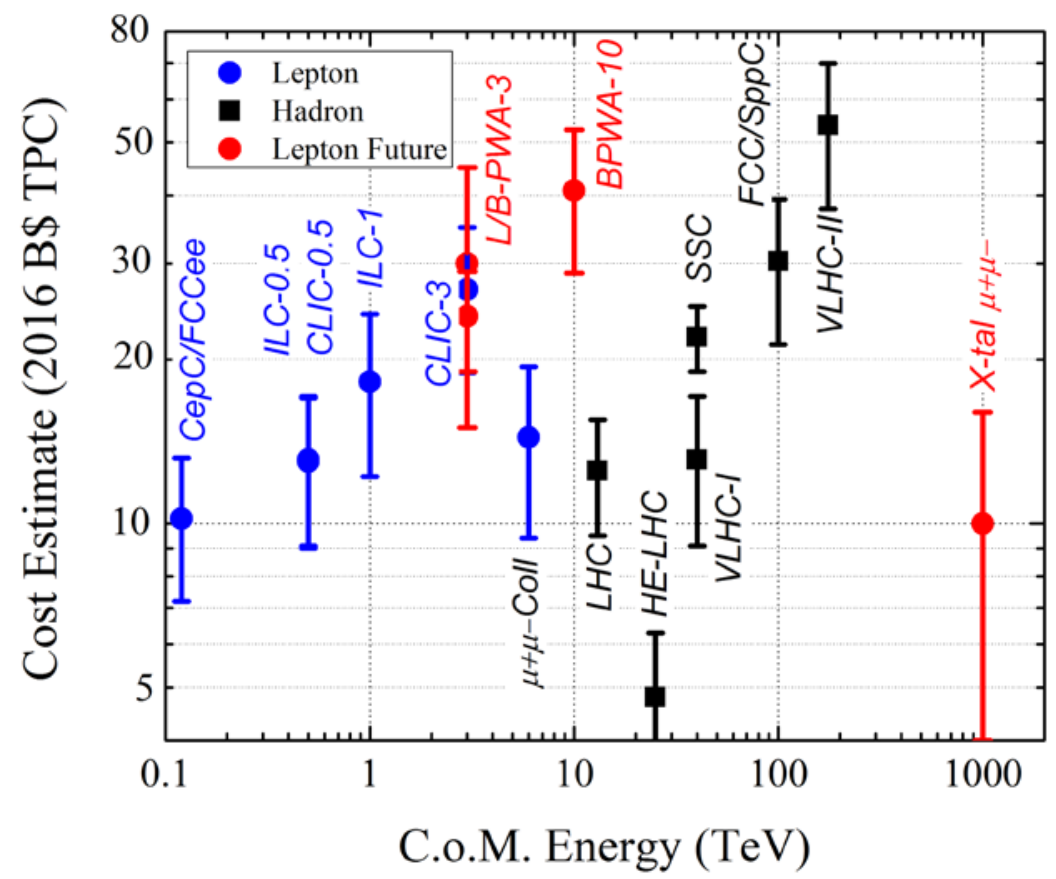

Fig. 1: Center-of-mass beam energy vs estimated Total Project Cost (TPC, the "US Accounting", in B\$) of various post-LHC frontier HEP accelerators: (blue dots) - lepton colliders, (black dots) - hadron colliders, (red dots) - future linear lepton colliders (see text).

One can see that only HE-LHC is certainly financially feasible being below the 10B\$ "feasibility level" discussed above. Several machines are potentially within the financial reach if undertaken as a global HEP project - CepC, FCC-ee, $0.5 \mathrm{TeV}$ options of ILC and CLIC, $6 \mathrm{TeV}$ c.o.m. muon collider and possibly, low-field option of the large circumference (233km) VLHCI. At the same time, really questionable seems to be the $1 \mathrm{TeV}$ version of the ILC, $3 \mathrm{TeV}$ CLIC, 87-km long SSC and 60-100 km FCC pp and SppC.

\section{Discussion: Ways to Proceed Toward Post-LHC Frontier HEP Facility}

Several ways to assure feasibility of future colliders are being considered. Firstly, significant savings can be acheioved by re-using the existing infrastructure and/or existing accelerators as injectors for the future ones. Of course, that can not be applied universally (eg., not for frontier leptonb colliders), but, for example, CERN's proton accelerator complex can be used in the FCC-pp or a muon collider. Secondly, launching extensive R\&D programs focused on the cost reduction of traditional technologies, e.g., SC magnets and tunneling, can greatly help, too [20]. At the same time, one should take into account that this approach has its limits. Thirdly, one could try to benefit significantly lower cost of doing business in Asia, particularly, in China - for example, comparison of modern synchrotron light sources shows a factor of about 3 lower construction cost for comparable facilities [20]. This advantage may or may not be in effect in the future but it definitely should be taken into account. The most promising option in the long run is to develop a new accelerator technology, namely, ultra-fast plasma wake-field acceleration (PWFA). The potential of the method is enormous, though recent attempts to design a collider 
based on laser- or beam-driven PWFA $[14,15]$ showed many serious not yet resolved issues such as modest average accelerating gradient $(\sim 2 \mathrm{GeV} / \mathrm{m}$ vs maximum single stage value of $10-50$ $\mathrm{GeV} / \mathrm{m}$ ), uncertain effectiveness of staging, low luminosity as the result of the beam emittance growth due to scattering of electrons and positrons in plasma, extremely tight tolerances on transverse and longitudinal stability of the collider elements, (currently) very low efficiency of the electric plug power conversion to beam power, etc. Cost-wise, such colliders are not very efficient at the present stage of development - see red dots in Fig. 1 and discussion in [17] - but, again, they have a significant potential for cost savings, for example, due to quick reduction of the cost per Watt of pulsed high power lasers.

Finally, one can try to assess options for "ultimate" future energy frontier collider facility with c.o.m. energies of 300-1000 TeV (20-100 times the LHC). We surely know that for the same reason the circular $e+e$ - collider energies do not extend beyond the Higgs factory range $(\sim 0.25$ $\mathrm{TeV}$ ), there will be no circular proton-proton colliders beyond $100 \mathrm{TeV}$ because of unacceptable synchrotron radiation power - they will have to be linear. It is also appreciated that even in the linear accelerators electrons and positrons become impractical above about $3 \mathrm{TeV}$ due to beamstrahlung (radiation due to interaction at the IPs) and about $10 \mathrm{TeV}$ due to radiation in the focusing channel $(<10 \mathrm{TeV})$. This leaves only $\mu+\mu$ - or $p p$ options for the "far future" colliders. If we further limit ourselves to affordable options and request such a flagship machine not to exceed $L_{f} \sim 10 \mathrm{~km}$ in length then we seek a new accelerator technology providing average gradient of $>30 \mathrm{GeV} / \mathrm{m}$ (compare with $E / L \sim 0.5 \mathrm{GeV}$ per meter in the LHC). There is only one such option known now: super-dense plasma as in, e.g., crystals [16], that excludes protons because of nuclear interactions and leaves us with muons as the particles of choice [2]. High luminosity can not be expected for such a facility if we limit the beam power and, with necessity, the total facility site power to some affordable level of $P \sim 100 \mathrm{MW}$. Indeed, as the energy of the particles $E$ grows, the beam current will have to go down at fixed power $I=P / E$, and, consequently, the luminosity will need to go down with energy. The paradigm shift from the past collider experience when luminosity scaled as $L \sim E^{2}$ will need to happen in the "far future" of HEP.

\section{Summary}

From the accelerator stand point, short answer to the question in the title of talk - "Will There Be Energy Frontier Colliders After LHC?" - is “may be”. Longer answer would include a notion that such a collider will first need a strong motivation for conctruction, i.e. dependent on the LHC results. If based on "traditional” accelerator technologies (SRF, SCMag, etc), only HELHC is cost feasible (will cost about half of that of the LHC), few others are close to the LHC TPC or exceed it slightly - CepC/FCCee, ILC, Muon Coll, VLHC-I, other proposed facilities would need either significant R\&D or use advantages of developing economy in China. The hopeful "non-traditional” technology of plasma acceleration is very expensive now, it needs sveral decades of R\&D to prove its feasibility as viable post-LHC collider option, on the other hand it has great potential and can be a basis of an "ultimate (dream)" $O(1 \mathrm{PeV})$ crystal muon collider which by necessity will be low luminosity and will require a paradigm change for HEP reaserch.

I greatly appreciate simulating discussions on these matters which I had at ICHEP2016 with K.Oide, V.Kozlov, P.Oddone, M.Breidenbach, M.Klein, A.Yamomote and M.Mangano. 


\section{References}

1. M.Tigner, A.Chao (Eds.), Handbook of Accelerator Physics and Engineering (World Scientific, Singapore, 1999)

2. V.Shiltsev, High energy particle colliders: past 20 years, next 20 years and beyond, Physics-Uspekhi $\mathbf{5 5 . 1 0}$ (2012) 965

3. B.Richter, High Energy Colliding Beams: What Is Their Future?, Rev. Acc. Sci.Tech. 7 (2014) 1

4. F.Zimmermann, High-energy physics strategies and future large-scale projects, NIMB 335 (2015) 4

5. P.Bhat, V.Shiltsev. Future HEP Accelerators: The US Perspective, arXiv:1511.00390

6. O.Brüning, S.Myers (eds.) Challenges and Goals for Accelerators in the XXI Century. World Scientific Publishing Company, 2016.

7. The International Linear Collider Technical Design Report, v.1: Executive Summary, eds. T.Behnke, et al, ILC-REPORT-2013-040 (2013)

8. CEPC-SPPC Preliminary Conceptual Design Report, The CEPC-SPPC Study Group, IHEP-CEPCDR-2015-01 (2015)

9. Future Circular Collider (FCC) http://cern.ch.fcc; also see M. Benedikt, F. Zimmermann, The Future Circular Collider Study, CERN Courier (28 March 2014)

10. J.P.Delahaye, The CLIC study of a multi-TeV linear collider, Annual Review of Nuclear and Particle Science 62 (2012) 105

11. S.Geer, Muon Colliders and Neutrino Factories, Annual Review of Nuclear and Particle Science $\mathbf{5 9}$ (2009) 347

12. O.Brüning, et al, High Energy LHC Document prepared for the European HEP strategy update. No. CERN-ATS-2012-237 (2012)

13. P.Limon (ed.), Design study for a staged very large hadron collider. Preprint FERMILAB-TM-2149 (2001)

14. Adli, E. et al. A beam driven plasma-wakefield linear collider: from Higgs factory to multi-TeV. In Electronic Proceedings of the Snowmass 2013 Community Study on the Future of High-Energy Physics arXiv:1308.1145

15. C.Schroeder, et al, Physics considerations for laser-plasma linear colliders. Physical Review Special Topics-Accelerators and Beams 13 (10) (2010), 101301

16. T.Tajima, M. Cavenago. Crystal X-ray accelerator, Physical Review Letters 59 (13) (1987) 1440

17. V.Shiltsev, A phenomenological cost model for high energy particle accelerators, JINST 9 (2014) T07002

18. LHC : The Guide, CERN-Brochure-2009-003 (2009)

19. V.Shiltsev, Crystal Ball: On the Future High Energy Colliders, PoS(EPS-HEP2015) 515 ; also in arXiv:1511.01934

20. V.Shiltsev, Accelerator physics and technology challenges of very high energy hadron colliders, Intl. Journ. Mod. Phys. A 30.23 (2015) 1544001 